Methods This study involved a randomly selected cross sectional survey of 2,058 households. Data were collected during home visits to all resident women of reproductive age (15-49 years). A geographic information system (GIS) was used to map all households and the only health centre in the district. The analysis was restricted to 2,206 rural children who were under the age of five years during the five years before the survey. Data were analysed using random effects Poisson regression.

Results $90.4 \%(1,996 / 2,206)$ of children lived more than 1.5 hours walk from the health centre. Children who lived $\geq 1$. 5 hrs from the health centre had a two-three fold greater risk of death than children who lived $<1.5$ hours from the health centre (children with travel time 1.5-<2.5hrs adjusted relative risk [adjRR] 2.3[0.95-5.6], travel time 2.5-<3.5hrs adjRR 3.1[1.3-7.4] and travel time 3.5$<6.5 \mathrm{hrs}$ adjRR 2.5[1.1-6.2]).

Conclusion Distance to a health centre had a marked influence on under five mortality in a poor, rural, remote area of Ethiopia. This study provides important information for policy makers on the likely impact of new health centres and their most effective location in remote areas.

\section{PS54 BIOMARKERS ASSOCIATED WITH MORTALITY IN A COMMUNITY BASED POPULATION WITH CARDIOVASCULAR RISK FACTORS: DATA FROM THE SCREENING TO PREVENT HEART FAILURE (STOP HF) STUDY}

doi:10.1136/jech-2012-201753.153

${ }^{1,2} \mathrm{CM}$ Conlon, ${ }^{1} \mathrm{CC}$ Kelleher, ${ }^{2} \mathrm{D}$ Dawkins, ${ }^{3} \mathrm{~L} \mathrm{McD}$ onald, ${ }^{2} \mathrm{M}$ Ledwidge, ${ }^{2,3 \mathrm{~K}} \mathrm{McD}$ Donald 'School of Public Health, Physiotherapy \& Population Science, University College Dublin, Dublin, Ireland; 'Heart Failure Unit, St Vincent's University Hospital, Dublin, Ireland, ${ }^{3}$ School of Medicine, University College Dublin, Dublin, Ireland

Background The STOP HF cohort is an ongoing longitudinal study of 1,040 individuals with cardiovascular risk factors and no known ventricular dysfunction at baseline. The study is located in South Dublin and the Southeast of Ireland. This is an analysis of the deaths reported at the interim analysis conducted in January 2012 (mean follow-up, 4 years, range 3.5-7.0 years).

Methods Univariable analyses compared the profiles of survivors to those deceased. Logistic regression identified the foremost associates of death. Cox's proportional hazard models estimated the risk of death over time, considering the presence of certain factors.

Results Between January 2005 and April 2008 1,040 individuals were recruited into the STOP HF study (Mean age 65, 49\% male, hyperlipidemia $53 \%$, hypertension $47 \%$, CAD 16\%, MI $8 \%$, arrhythmia $8 \%$, diabetes $12 \%$, smoker $14 \%$ ). At the interim analyses 64 deaths were reported, (6.1\% of the cohort). Univariable analyses indicated those deceased were older ( $65 \mathrm{vs.} 71$ years, $p<0.0001)$ with lower diastolic blood-pressure $(p<0.005)$ and a $17 \%$ greater prevalence of diabetes $(p<0.0001)$. Those deceased had biomarker levels more frequently $\geq 75$ th percentile for gender in BNP $(p<0.005)$, hsCRP $(p<0.0001)$, Urea $(p<0.005)$, Creatinine $(p<0.0001)$ and ALP $(p<0.05)$ and were more likely to be taking ACE inhibitor $(p<0.02)$ and anti-diabetic therapy $(p<0.0001)$. The final multivariable model identified age $(\operatorname{ExpB}=1.07, \mathrm{OR} 1.03-1.11, \mathrm{p}<0.0001)$, diabetes $(\operatorname{ExpB}=3.44, \mathrm{OR} 1.96-7.00, \mathrm{P}=0.001), \mathrm{BNP}(\operatorname{ExpB}=1.00$, OR 1.0001.007, $\mathrm{p}=0.075)$ and hsCRP $(\operatorname{ExpB}=1.07, \mathrm{OR} 1.04-1.11, \mathrm{p}<0.000)$ as associates of mortality (model $\chi 2(4, \mathrm{~N}=1041)=50.240, \mathrm{p}<0.001$ ). Controlling for age, cox's proportional hazard models indicated that those with diabetes had a 3 times higher risk of death $(\operatorname{ExpB}=3.117$, OR 1.801-5.304, $\mathrm{p}<0.0001$ ); those with a hsCRP level $\geq 75$ th percentile for gender were almost 3.5 times more likely to die $(\operatorname{ExpB}=3.439$, OR 1.995-5.498, $\mathrm{p}<0.0001$ ) and those with ALP $\geq 75$ th percentile for gender were also more likely to die durin the follow-up period (ExpB=1.919, OR 1.096-3.359).

Conclusion Diabetes is associated with in excess of a 3 times increase in the likelihood of death over 4 years follow-up in this population, while other morbid conditions such as coronary disease and atrial fibrillation show no significant association. Despite aggressive cardiovascular risk management through primary and secondary prevention strategies, those with diabetes continue to have a poor prognostic outlook. Observation of biomarkers such as hsCRP and ALP may be of added value in monitoring cohorts with existing cardiovascular risk factors and identifying those that may be subclinically manifesting heightened risk of mortality in this setting.

\section{PS55 THE USE OF A WEALTH INDEX WITHIN AN IMPOVERISHED COMMUNITY: A COHORT STUDY IN KWAZULU-NATAL, SOUTH AFRICA}

doi:10.1136/jech-2012-201753.154

1,2LL Davidson, ${ }^{3} \mathrm{~S}$ Kauchali, ${ }^{3} \mathrm{MK}$ Chhagan, ' $\mathrm{F}$ Bah, ${ }^{200 T}$ Uwemedimo, ${ }^{3} \mathrm{MH}$ Craib, ${ }^{4}$ McKeague. 'Epidemiology, Columbia University, New York City, USA;²Pediatrics, Columbia University, New York City, USA; ${ }^{3}$ Child Health, University of KwaZulu-Natal, Durban, South Africa; ${ }^{4}$ Biostatistics, Columbia University, New York, USA

Background An index of wealth is widely used in national surveys to create economic profiles. We constructed such an index within a population-based cohort study of 1,788 preschool children and their primary caregivers living in five isiZulu tribal areas of KwaZuluNatal, South Africa, an extremely poor area which remains at the epi-center of the South African HIV/AIDS epidemic.

Methods Study Design: Information on household assets, employment, household structure was obtained by door-to-door survey alongside a screen for child disability in developing countries. All children were invited to a structured medical and psychological assessment for disability and HIV status of child and primary caregiver. Wealth Index: A household asset index developed within our study population used many items identical to those in WHO DHS surveys, employing a principal components approach. Asset indicators were grouped into 3 categories: land ownership, ownership of consumer goods, and characteristics of household dwelling (building material, water sources, toilet facility, energy source, etc.). Factor analysis was performed: variables with zero variances and all variables with prevalence less than $2 \%$ were removed. The first component explained $16 \%$ of the variance and a $\mathrm{KMO}$ of 0.532 . We ranked the factor scores on the first component in ascending order, standardized to a range of 0 to 4 , and grouped into tertiles ( 1 =lowest third, $2=$ middle third, $3=$ top third).

Results The wealth index gave information about study participation: Of 1788 children screened, children from households in the poorest third were significantly more likely to be brought to the assessment: $91 \%$ compared to 83 and $89 \%$ in the middle and top third respectively. Significant differences in the wealth index were found between the five areas in the sample. With regard to caregiver responses about child disability, those in the poorest third were most likely to report that their child had a disability (43\% compared to 46 and $49 \%$ - significant on test for trend). Though doctors found a lower rate of disability than reported by parents, there was no difference in disability or false positives rate by wealth index tertile HIV seropositivity of caregiver varied by tertile $(30.3 \%, 28.5 \%$ and $21.8 \%$ in poorest, middle and least poor) but not that of children. Conclusion Even within extremely deprived areas in a low or middle income country, a wealth index can assess comparative risk among groups suffering more or less material disadvantage and also provide important information in assessing possibility of selection bias in the findings.

\section{PS56 RISK OF INTENTIONAL SELF-HARM IN YOUNG PEOPLE WITH SELECTED MENTAL AND CHRONIC PHYSICAL CONDITIONS IN ENGLAND}

doi:10.1136/jech-2012-201753.155

0 Seminog, M Goldacre. Department of Public Health, University of Oxford, Oxford, UK 
Background Self-harm is a well recognised act of psychological distress in some young people. Children and young adults with chronic disease are known to have an increased risk of psychological distress, but their risk of intentional self-harm is not well documented in England. We aimed to determine the risk of intentional self-harm in an English population aged 10-24 years with a range of psychological disorders, namely schizophrenia, bipolar disorder, anorexia/bulimia, depression and in those with chronic medical conditions, including diabetes mellitus, asthma and epilepsy.

Methods We used a dataset of English national linked Hospital Episode Statistics for 1999 - 2011. We identified all the records of people who were admitted to the National Health Service (NHS) hospitals with these conditions. Each condition-specific cohort was constructed by identifying the first admission for the "exposure" condition in people aged 10 to 24 years, and we analysed the dataset for any subsequent admission for intentional self-harm. The rate of self-harm in each exposure cohort was compared with that in a reference cohort comprising people admitted for a wide range of minor medical and surgical conditions; and expressed as a rate ratio (RR) with $95 \%$ confidence intervals (CI).
Results The RR (95\% CI) for intentional self-harm was significantly and substantially high in people with schizophrenia 5.93 (5.7 to 6.17), bipolar disorder 7.93 (7.26 to 8.64), anorexia/bulimia 5.29 (4.97 to 5.63), and depression 10.25 (9.95 to 10.57). It was also significantly high, though much less so, in people with diabetes 1.60 (1.52 to 1.68 ), asthma 1.52 (1.48 to 1.56 ) and epilepsy 1.88 (1.80 to 1.96). These rates remained elevated after we excluded the cases of self-harm in the first year following hospitalisation with each exposure condition.

Conclusion Children and young adults with the psychiatric conditions studied are at substantially increased risk of intentional selfharm. People with the chronic medical diseases studied were also at increased risk, although not nearly as high as that associated with the psychiatric disorders. The elevated risks were maintained more than a year after admission, and are likely to be associated with the disorder rather than the fact of hospital admission. Self-harm is important not only for the distress of the act itself, but it is also recognised as a predictor for eventual suicide. Our findings suggest that current suicide prevention programmes should consider targeting these risk groups as a priority. 